\title{
Estudo retrospectivo da utilização do transplante de membrana amniótica em um serviço terciário de Oftalmologia
}

\author{
Retrospective study of amniotic membrane transplantation \\ in a terciary ophthalmic health care service
}

Eduardo Conforti de Oliveira', Gabriel Zatti Ramos², Vivian Cristina Costa Afonso', Luciene Barbosa Souza

\section{ResUMO}

Objetivo:Nos últimos anos , o transplante de membrana amniótica (MA) tem sido utilizado com freqüência na reconstrução da superfície ocular em diversas doenças oculares. Este estudo objetiva identificar as indicações mais freqüentes do transplante de MA, assim como avaliar os resultados deste procedimento. Métodos: Foram analisados retrospectivamente os prontuários de 107 pacientes submetidos ao transplante de MA, avaliando a indicação do transplante de MA e a evolução do quadro no período pósoperatório. Resultados: Dos 107 pacientes estudados, as indicações de MA mais freqüentes foram exérese de pterígio $33.64 \%(\mathrm{n}=36)$, defeito epitelial persistente $29.90 \%(n=32)$, liberação de simbléfaro $18.69 \%(n=20)$, queimadura química $6.54 \%(\mathrm{n}=7)$, cérato-conjuntivite primaveril $4.67 \%(\mathrm{n}=5)$, síndrome de Stevens-Johnson $2.80 \%(n=3)$, penfigóide ocular cicatricial $1.86 \%(n=2)$, ceratopatia bolhosa $1.86 \%(n=2)$. No seguimento dos pacientes houve recidiva do pterígio em (6.25\%) nos pterígios primários e em $(15 \%)$ nos pterígios recidivados. Não houve recidiva nos pacientes com simbléfaro, tendo como causa pterígio recidivado. Houve fechamento do epitélio com o uso de MA nos pacientes do estudo variando de 50 a $80 \%$, dependendo da doença de base. Na fase aguda da queimadura química houve fechamento epitelial em $50 \%$ dos casos. Já nos que utilizaram MA na fase crônica da queimadura a restauração da superfície ocular ocorreu em $33 \%$ dos casos. Uso da MA na cérato-conjuntitivite primaveril com papilas gigantes, $80 \%$ não apresentaram recidiva das papilas.Todos os pacientes que tiveram indicação de MA, devido à ceratopatia bolhosa apresentaram diminuição da dor. Os pacientes que tiveram como causa da utilização de membrana a Síndrome de Stevens-Johnson (SSJ) na fase aguda apresentaram diminuição importante do processo inflamatório . Dos pacientes que receberam enxerto de MA na fase crônica da SSJ, 50\% apresentaram evolução favorável. Conclusão: $\mathrm{O}$ enxerto de membrana amniótica nas diferentes doenças avaliadas apresentou resultados favoráveis na maioria delas. Esta técnica pode ser considerada como boa alternativa terapêutica a ser empregada nestas situações, além de ser um método barato e de fácil execução cirúrgica.

Descritores: Âmnio/transplante; Procedimentos cirúrgicos reconstrutivos/tendências; Transplante de tecidos ; Oftalmopatias/cirurgia ; Doenças da túnica conjuntiva/cirurgia; Doenças da córnea/cirurgia; Estudos retrospectivos

\footnotetext{
${ }^{1}$ Médico assistente do Hospital Oftalmológico de Sorocaba - HOS - Sorocaba (SP),Brasil;

Estagiários do Departamento de Retina do Hospital Oftalmológico de Sorocaba - HOS - Sorocaba (SP), Brasil;

${ }^{3}$ Coordenadora do Serviço de Residência Médica do Hospital Oftalmológico de Sorocaba - HOS - Sorocaba (SP), Brasil; Professora do Programa de Pós-Graduação em Oftalmologia da Universidade Federal de São Paulo - UNIFESP - São Paulo (SP), Brasil.
} 


\section{INTRODUÇÃO}

$\mathbf{N}$ os últimos anos, o transplante de membrana amniótica (MA) tem sido utilizado com freqüência na reconstrução da superfície ocular em doenças cicatriciais da córnea e conjuntiva como queimaduras químicas, penfigóide ocular cicatricial, eritema multiforme, dentre outras ${ }^{(1.3)}$.

A membrana amniótica promove cicatrização nos defeitos epiteliais persistentes por facilitar a adesão e migração de células epiteliais basais, além de prevenir a apoptose celular e restaurar o fenótipo epitelial ${ }^{(4)}$,também tem ação angiogênica, cicatricial, antinflamatória e antimicrobiana ${ }^{(1,4,6)}$.

A MA tem sido usada para tratamento de diversas doenças como: úlcera de perna, perda de pele, síndrome de Stevens-Jhonson, cirurgias pélvicas e vaginais, queimaduras de pele e cicatrizes cirúrgicas ${ }^{(7)}$. Seu primeiro uso em oftalmologia foi em 1940 para tratamento de defeito conjuntival ${ }^{(7)}$. Recentemente tem sido utilizado também para tratamento de doenças cicatriciais da conjuntiva, queimaduras químicas e defeitos epiteliais persistentes ${ }^{(7)}$. Ela possui diversas propriedades incluindo efeitos antiadesivos, antibacterianos, proteção de lesões, redução da dor e facilitador de epitelização ${ }^{(7,8)}$.

A MA é composta principalmente por uma camada espessa de colágeno, membrana basal de laminina e colágeno tipo IV. É considerada imunologicamente inerte por não expressar antígenos de histocompatibilidade (HLA-A,B ou DR), conseqüentemente não induzindo rejeição imunológica ${ }^{(7)}$. A MA, especialmente o epitélio, também produz vários fatores de crescimento, incluindo fator de crescimento basal de fibroblasto, fator de crescimento de hepatócito e fator de crescimento de transformação beta. Esses fatores de crescimento podem modular a proliferação e diferenciação de células corneanas e conjuntivais ${ }^{(7)}$.

Este estudo tem como objetivo analisar as indicações mais freqüentes do transplante de MA em um serviço de referência em oftalmologia, bem como avaliar os resultados deste procedimento.

\section{Métodos}

Foram analisados retrospectivamente os prontuários de 107 pacientes submetidos a transplante de MA no Hospital Oftalmológico de Sorocaba, no período de janeiro de 2005 a janeiro de 2007. A membrana amniótica foi obtida de placentas provenientes de cesáreas eletivas de pacientes da Santa Casa de Sorocaba.Todas essas pacientes tinham exames sorológicos negativos para HIV, Hepatite B, Hepatite C e Sífilis, reconfirmados pela sorologia do sangue do cordão umbilical depois do parto. A MA foi obtida no centro cirúrgico obstétrico; e procedeu-se sua lavagem com soro fisiológico $0.9 \%$ em ambiente estéril e a seguir à lavagem com solução tampão fosfato.

Realizada a adequada preparação, MA foi estendida sobre papel de filtro de nitrocelulose estéril , com a face epitelial para cima. A MA aderida ao filtro foi cortada em fragmentos que variaram de 2 a $4 \mathrm{~cm}^{2}$, colocada em um recipiente estéril contendo glicerol e meio de preservação de córnea TC-199 na proporção 1:1. Posteriormente era congelada a $-80^{\circ} \mathrm{C}$, seguida de refrigeração a $4^{\circ} \mathrm{C}$. O tempo máximo de utilização das membranas foi de 2 meses de congelamento..

As cirurgias foram realizadas pela mesma equipe, sendo a membrana fixada com fio nylon $10-0 \mathrm{em}$ pontos separados e utilizada com a face epitelial para baixo somente nas queimaduras agudas; nas demais indicações a membrana foi fixada com sua face epitelial para cima.

Para tratamento de Defeito Epitelial Persistente, tendo como causa queimadura química, foi realizado transplante de células limbares juntamente com o enxerto de membrana amniótica, nas demais causas foi realizado apenas o enxerto de MA.

No pós-operatório foi realizado acompanhamento de acordo com o grupo a ser estudado.Nos grupos, (exérese de pterígio, liberação de simbléfaro e Síndrome de Stevens Johnson) os pacientes foram acompanhados nos primeiro, sétimo,trigésimo dias, 3 e 6 meses, 1 ano, 1,5 anos de cirurgia, nos grupos (Defeito epitelial persistente, Queimadura química, Cerato-conjuntivite primaveril ) foram avaliados no primeiro, sétimo,décimo quinto e trigésimo dias e 1,5, 3 e 6 meses e 1 ano de cirurgia, no grupo de ceratopatia bolhosa os pacientes foram acompanhados no primeiro,sétimo,trigésimo dias e terceiro mês de cirurgia.

Avaliou-se também neste estudo, dados dos prontuários referentes às doenças que originaram a indicação do transplante de membrana amniótica e a evolução do quadro no pós-operatório.

\section{Resultados}

Dos 107 pacientes nos quais foi feito uso de membrana amniótica, encontramos as seguintes indicações 
em ordem de freqüência: exérese de pterígio $33.64 \%(n=36)$, defeito epitelial persistente (DEP) $29.90 \%(n=32)$, liberação de simbléfaro $18.69 \%(n=20)$ , queimadura química $6.54 \%(n=7)$, cérato-conjuntivite primaveril $4.67 \%(n=5)$, síndrome de Stevens-Johnson $2.80 \%(n=3)$, penfigóide ocular cicatricial $1.86 \%(n=2)$ e ceratopatia bolhosa $1.86 \%(n=2)$ (Gráfico 1$)$.

Do grupo de pacientes nos quais se utilizou MA isoladamente (exérese de pterígio), 44,44\% ( $\mathrm{n}=16)$ eram pterígios primários e $55,56 \%(\mathrm{n}=20)$ pterígios recidivados . Esses pacientes foram avaliados em um ano e meio e foi observado recidiva do pterígio em $6.25 \%(n=1 / 16)$ dos casos em exérese de pterígios primários. Recidiva de $15 \%(n=3 / 20)$ nos casos pósexérese dos pterígios recidivados foi encontrada.

No grupo de pacientes no qual foi feito uso de MA por DEP $(n=32)$, as principais causas desta doença, em ordem de frequência foram: pós-cirurgia de transplante de córnea $31.25 \%(n=10)$, pós-ceratite herpética $21.80 \%(n=7)$, retransplante córnea pós-transplante tectônico $18.75 \%(n=6)$, pós-queimadura química $15.62 \%(n=5)$, pós-ceratite bacteriana $6.25 \%(n=2)$ e por artrite reumatóide $6.25 \%(\mathrm{n}=2)$. Esses pacientes foram acompanhados $1^{\circ}, 7^{\circ}, 15^{\circ}, 30^{\circ}$ e 45 dias , $3^{\circ}$ e $6^{\circ}$ meses de enxerto da MA, observando-se o fechamento do epitélio, com a reabsorção da MA nos pacientes que utilizaram MA em: $80 \%(n=4 / 5)$ pós-queimadura química, $70 \%(\mathrm{n}=7 / 10)$ pós-transplante de córnea, 57.14\% $(n=4 / 7)$, pós-ceratite herpética, $50 \%(n=1 / 2)$, pósceratite bacteriana, $50 \%(\mathrm{n}=3 / 6)$ pós-retransplante, pós-transplante tectônico, $50 \%(\mathrm{n}=1 / 2)$ por artrite reumatóide.

Os pacientes que utilizaram membrana amniótica pós-liberação de simbléfaro $(\mathrm{n}=20)$ tiveram como etiologia deste sinal: pós-queimadura química $60 \%(n=12)$, pterígio recidivado $30 \%(n=6)$, síndrome Stevens-Johnson (seqüela) 10\% $(n=2)$, esses pacientes foram reavaliados um ano e meio da cirurgia de enxerto de MA apresentando recidiva do simbléfaro em: $16.6 \%(n=2 / 12)$ pós-queimadura química, $50 \%(n=1 / 2)$ na Síndrome de Stevens-Johnson. Não foi observada recidiva do simbléfaro nos pacientes que tiveram como indicação de uso da MA pósliberação de simbléfaro tendo como causa pterígio recidivado.

Nos pacientes que utilizaram enxerto de MA devido à queimadura química $(\mathrm{n}=7), 57.14 \%(\mathrm{n}=4)$ utilizaram membrana na fase aguda da queimadura e $42.85 \%(n=3)$ utilizaram na fase crônica para promo-

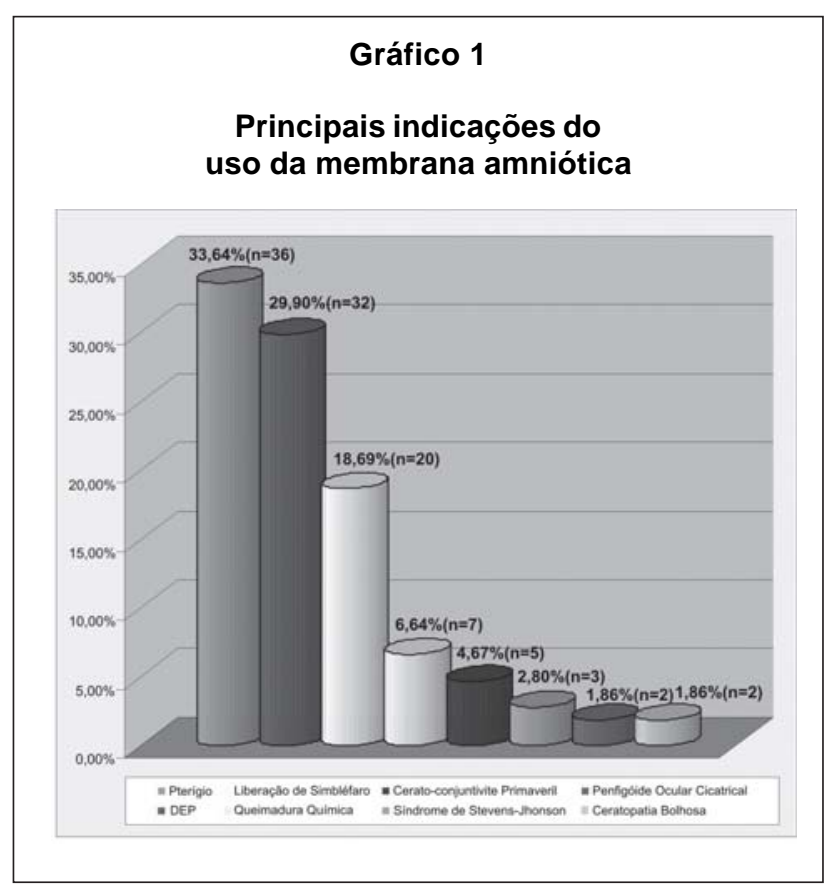

ver reconstrução da superfície ocular (nestes foi necessário usar conjuntamente enxerto de limbo do olho contralateral, pois apresentavam insuficiência limbar). Os pacientes que utilizaram a MA na fase aguda foram acompanhados $1^{\circ}, 07^{\circ}, 15^{\circ}, 30^{\circ}$ e 45 dias $3^{\circ}$ e $6^{\circ}$ mês da cirurgia apresentando fechamento epitelial em $50 \%$ dos casos. Já os que utilizaram MA na fase crônica foram acompanhados até um ano da cirurgia apresentando restauração da superfície ocular em 33\%(n=1/ 3) dos casos.

$\mathrm{O}$ grupo de pacientes que utilizaram membrana amniótica, tendo como causa da indicação cératoconjuntitivite primaveril $(n=5)$, eram pacientes crônicos que apresentavam papilas gigantes na conjuntiva tarsal superior não responsivas ao tratamento clínico e com repercussões corneais. Nesses casos, foi realizado a exérese das papilas com lâmina 15.0 e implante da membrana amniótica na conjuntiva tarsal superior com face estromal da membrana voltada para conjuntiva tarsal. Esses pacientes foram acompanha$\operatorname{dos} 01^{\circ}, 07^{\circ}, 15^{\circ}, 30^{\circ}$ e $45^{\circ}$ dias e nos , $3^{\circ}$ e $6^{\circ}$ meses e 1 ano de cirurgia, com $80 \%(n=4)$ não apresentando recidiva das papilas e $20 \%(n=1)$ apresentaram recidiva das papilas.

Dos pacientes que tiveram como causa da utilização de membrana a Síndrome de StevensJohnson, $(n=3)$ esta foi utilizada na fase aguda em $33,33 \%(n=1)$ e na fase crônica em $66,66 \%(n=2)$. O 
paciente que recebeu enxerto de membrana amniótica na fase aguda foi acompanhado nos dias 07, 15 e 45 dias do período pós-operatório e apresentou diminuição importante do processo inflamatório ativo. Já os pacientes que receberam enxerto de membrana amniótica na fase crônica foram acompanhados 15, 30 dias, $3^{\circ}$ e $6^{\circ}$ meses e 1 ano da intervenção, sendo que $50 \%(n=1)$ destes casos apresentaram reatividade do processo inflamatório ativo trinta dias pós-enxerto e $50 \%(\mathrm{n}=1)$ apresentaram evolução favorável para futuras cirurgias de restauração ocular, sem recidiva do simbléfaro.

Os pacientes que tiveram indicação de MA devido à ceratopatia bolhosa $(\mathrm{n}=2)$ foram acompanhados $01^{\circ}, 07^{\circ}, 30$ dias e 3 meses, demonstrando diminuição da dor em $100 \%$ das vezes.

Os pacientes com penfigóide ocular cicatricial perderam o seguimento em nosso serviço.

\section{Dıscussão}

\section{O uso de membrana amniótica e suas características}

O transplante de MA pode ser considerado uma alternativa para diversas situações, mostrando-se um excelente substrato para reconstrução da superfície conjuntival e também em algumas afecções corneanas $^{(9)}$.

Nos casos de queimadura química em que há comprometimento severo da região limbar ou posteriormente ocorre a formação de simbléfaro, o uso da MA tem apresentado bons resultados quando utilizado como substrato para reconstrução da região alterada ${ }^{(9)}$. Nas queimaduras químicas na fase aguda pode-se utilizar a MA como lente de contato utilizando seu epitélio para baixo objetivando-se efeito antiinflamatório, promover rápida epitelização, diminuição da dor e prevenção para o desenvolvimento de seqüelas a longo prazo ${ }^{10}$. Pode ser utilizada também na fase crônica na reconstrução da superfície ocular ${ }^{(9-11)}$.

Em pequenas queimaduras o enxerto de membrana amniótica rapidamente restaura as superfícies corneana e conjuntival, e em queimaduras severas há certa restauração da superfície ocular conjuntival, com debilitação da formação de simbléfaro e redução da inflamação estromal e limbar, porém não previne deficiência das células germinativas limbares ${ }^{(10)}$. O uso de membrana amniótica como terapêutica isolada não é suficiente para promover a cura corneana na ausência de células germinativas limbares ${ }^{(12)}$. Os resultados da atual pesquisa não foram tão satisfatórios quanto os apresentados por Shimazaki et al. ${ }^{(13)}$ que apresentou altíssima incidência da restauração epitelial em seus pacientes que foram submetidos a enxerto de membrana amniótica pós-queimadura química ou térmica.

Na presente pesquisa, observou-se ótimos resultados do enxerto de membrana amniótica nos pacientes com ceratoconjuntivite primaveril, $80 \%$ não apresentaram recidiva das papilas e nos $20 \%$ restantes apesar da recidiva papilar encontrada, esta era muito mais branda que o quadro clínico anterior e não traziam repercussão corneana.

Tseng et al. ${ }^{(14)}$ sugerem o uso de membrana amniótica para reconstrução da superfície conjuntival, estando seu índice de sucesso relacionado com a quantidade de tecido conjuntival sadio remanescente, e extensão corneana sem deficiência de células germinativas limbares.

O uso de membrana amniótica pode ser considerado uma boa alternativa no tratamento cirúrgico de pterígio, especialmente nos pterígios primários porque sua taxa de recorrência é significativamente menor se comparado com aproximação conjuntival direta $^{(15)}$ e transplante conjuntival. Também, o uso da MA pode ser indicado para os casos em que há envolvimento conjuntival difuso (pterígio nasal e temporal), e naqueles casos onde se deseja preservar a conjuntiva bulbar para uma futura cirurgia filtrante de glaucoma ${ }^{(15)}$. Em estudo realizado por Ma et al ${ }^{(16)}$ observou-se a eficácia e a segurança do enxerto de MA, como terapia adjuvante após a remoção de pterígio primário, comparado com enxerto conjuntival e instilação de mitomicina tópica; foi observado que não houve diferença significante na taxa de recorrência do pterígio entre os 3 grupos estudados. Os resultados do atual trabalho quanto à utilização de membrana amniótica pós-liberação de simbléfaro, tendo como causa do simbléfaro pterígio recidivado, assemelham-se aos descritos por Shimazaki et al. ${ }^{(7)}$ não observando-se recidiva do simbléfaro no seguimento, porém em seu estudo foi utilizado limbo autólogo juntamente com a membrana amniótica e nós apenas utilizamos o enxerto de membrana.

A MA também pode ser utilizada no tratamento de defeitos epiteliais persistentes, especialmente nos casos refratários ao tratamento convencional em que há comprometimento mais severo das células limbares, onde geralmente é necessário transplante de células limbares .O uso da membrana amniótica aumenta a 
taxa de sucesso do tratamento pois ajuda na reconstrução do estroma perilimbal e reduz a inflamação e vascularização no local ${ }^{(14)}$. Ela possui uma membrana basal espessa e matriz estromal avascular, a membrana basal facilita a migração, adesão e diferenciação das células epiteliais basais sendo também importante para prevenir a apoptose epitelial. Essas ações explicam porque a membrana amniótica efetivamente permite a rápida epitelização corneana ${ }^{(17)}$. Além disso, a membrana basal da membrana amniótica funciona como substrato para o crescimento das células epiteliais germinativas, prolongando seu período de vida e mantendo sua capacidade de produzir colônias (clonogenicidade), explicando o mecanismo de expansão das células germinativas do limbo e células amplificadoras transitórias da córnea durante o tratamento da deficiência límbica parcial ${ }^{(11-14)}$.

Chen et al. ${ }^{(18)}$ utilizou transplante de MA para tratamento de úlcera neurotrófica grave e foi observado em seu estudo cicatrização da úlcera em 76.4\% ( $n=13 / 17)$ em 16.6 dias pós-enxerto de MA. Nessa atual pesquisa, foi observado fechamento do epitélio corneano pós-enxerto de MA para tratamento de DEP em: $80 \%(n=4 / 5)$ dos casos pós-queimadura química, 70\% (n=7/10) pós-transplante de córnea, 57.14\% $(n=4 / 7)$ pós-ceratite herpética, 50\% $(n=1 / 2)$ pósceratite bacteriana, $50 \%(n=3 / 6))$ pós-retransplante de córnea pós-transplante tectônico e $50 \%(n=1 / 2)$ pósartrite reumatóide.

O penfigóide ocular cicatricial e a síndrome de Stevens-Johnson são doenças auto-imunes, caracterizadas por inflamação crônica recorrente, podendo causar conjuntivite cicatricial, olho seco severo, disfunção de células germinativas conjuntivais corneanas, rejeição de transplante de córnea, meibomite e triquíase severas. A utilização de membrana amniótica é importante na reconstrução da superfície ocular tanto como novo substrato para conjuntiva bulbar, como também para procedimento inicial antes da realização de transplante de limbo ${ }^{1}$. Na síndrome de Stevens-Johnson pode-se usar membrana amniótica durante a fase aguda da doença quando há risco de perfuração, realizando-se um recobrimento corneano e também na fase crônica da doença tentando-se promover membrana basal ${ }^{(1)}$. Podese também com seu uso conseguir uma boa restauração da superfície bulbar e boa profundidade do fórnice e prevenir a recorrência de simbléfaro em caso severos ${ }^{(19)}$.É necessário lembrar que as defesas da superfície ocular sejam restauradas prévia ou simultanea- mente a reconstrução da superfície ocular pelo enxerto de MA com ou sem transplante de células germinativas do limbo ${ }^{(11)}$.Incluem nessas medidas a oclusão do ponto lacrimal ou o uso de soro autólogo para deficiência lacrimal severa ${ }^{(1)}$, correção plástica da margem palpebral quando necessário e problemas envolvendo cílios, tarsorrafia para evitar exposição ocular e outros. No presente estudo, observou-se recidiva de simbléfaro em $50 \%$ dos pacientes que utilizaram membrana amniótica pós-liberação do simbléfaro na fase crônica da síndrome de S. Johnson diferindo do estudo apresentado por Honavar et al. ${ }^{(19)}$ onde não foi observado recidiva de simbléfaro no seguimento de seus pacientes.

Em pacientes com ceratopatia bolhosa o recobrimento corneano com membrana amniótica é uma opção para alivio da dor. O efeito benéfico da membrana amniótica na ceratopatia bolhosa pode ser atribuído a adesão de um novo substrato composto pala membrana basal e matriz estromal avascular da membrana amniótica, além disso apresenta propriedades estimuladoras de epitelização e moduladoras de processos cicatriciais e inflamatórios ${ }^{(20)}$. Resultados obtidos em estudo realizado pela Unifesp-EPM ${ }^{(21)}$ assemelham-se aos descritos por Pires et al. ${ }^{(20)}$, onde observouse ausência de dor em $90 \%$ dos pacientes com ceratopatia bolhosa submetidos a esse procedimento. Na presente casuística, limitou-se apenas a 02 pacientes submetidos a esse procedimento com diminuição da dor em $100 \%$ deles. Seu efeito é temporário, pois ela é reabsorvida em aproximadamente duas semanas (7). Porém, apesar da reabsorção precoce da membrana, observou-se seu efeito "analgésico" até o término do seguimento.

\section{Conclusões}

As indicações mais freqüentes de transplante de MA no serviço de referência em oftalmologia do Hospital Oftalmológico de Sorocaba foram: exérese de pterígio, defeito epitelial persistente e liberação de simbléfaro.

Os melhores resultados obtidos com o transplante da MA foram a redução da dor em $100 \%$ dos casos de ceratopatia bolhosa, ausência de recidiva de simbléfaro nos pacientes que tiveram como causa base o pterígio recidivado, ausência de recidiva de papilas na conjuntiva tarsal superior em $80 \%$ dos pacientes com ceratoconjuntivite primaveril, fechamento 
epitelial em $80 \%$ dos pacientes que apresentavam defeito epitelial pós-queimadura química.

O enxerto de membrana amniótica nas diferentes doenças avaliadas apresentou resultados favoráveis na maioria delas. Esta técnica pode ser considerada uma boa alternativa terapêutica a ser empregada nestas situações, além de ser um método barato e de fácil execução cirúrgica.

Contudo, novos estudos com maior número de pacientes em cada grupo estudado são necessários para elucidar melhor a importância da MA no tratamento das diferentes doenças oculares.

\section{Abstract}

Objective: During the last years amniotic membrane (AM) autograft has been frequently used for reconstruction of the ocular surface in many diseases. This study intends to analyze the major indications for AM as well the results of this procedure. Methods: Data of 107 patients who had done AM autograft surgery were retrospectively analised regarding the indications and postoperatory follow-up. Results: The indications for AM autograft was ptherygium 33.64\% ( $n=36)$, persistent epithelial defect $29.90 \% \quad(n=32)$, simblepharum $18.69 \%(n=20)$, chemical injury $6.54 \%(n=7)$, vernal conjunctivitis $4.67 \%(n=5)$, StevensJohnson $2.80 \%$ ( $n=3$ ), ocular cicatricial penfigoid $1.86 \%$ $(n=2)$, bullous keratopathy $1.86 \%(n=2)$. At the followup of ptherygium was observed recurrence between $6,25 \%$ (in primary ptherygium) and $15 \%$ (in recurrence ptherygium). No recurrence was observed in simblepharum surgery for complications due ptherygium. Recovery of epithelial integrity was between 50 and $80 \%$ depending the disease. In the acute phase of chemical burns recovery was observed in $50 \%$, however in chronic chemical burns the recovery was only in $33 \%$ of the cases. The AM in vernal conjunctivitis to resolve giant papillae, $80 \%$ of the cases showed good evolution. In $100 \%$ of the case due bullous keratopathy the patients had improvement of the pain. In Steven-Johnsons syndrome (SJS) at the acute phase the patients had improevment of the inflammatory component. In the chronic phase of SJS, $50 \%$ of the patients had favorable evolution after the procedure. Conclusion: Amniotic membrane autograft showed promising results for the majority ocular surface diseases enrroled in this study. This surgical tecnic cheap and easy doing asweel a good therapeutic alternative for many ocular surface diseases.
Keywords: Amnio/transplantation; Reconstructive surgical procedures /trends; Tissue transplantation; Eye diseases/surgery; Conjunctival diseases/surgery; Corneal diseases/surgery; Retrospective studies

\section{REFERÊNCIAS}

1. Tsubota K, Satake Y, Ohyama M, Toda I, Takano Y, Ono M, et al. Surgical reconstruction of the ocular surface in advanced ocular cicatricial pemphigoid and Stevens-Johnson syndrome.Am J Ophthalmol 1996;122(1):38-52.

2. Gomes JAP, Komagome CM, Santos N, Cunha MC, Freitas D. Membrana amniótica nas cirurgias reconstrutivas da superfície ocular nas ceratoconjutivites cicatriciais. Arq Bras Oftalmol 1999; 62(5):562-76.

3. Gomes JAP. Atualização no tratamento das ceratoconjutivites cicatriciais . Arq Bras Oftalmol. 2000;63(1):91-6.

4. Kim JC, Tseng, SC. Transplantation of preserved human amniotic membrane for surface reconstruction in severely damaged rabbit corneas . Cornea 1995 ;14(5):473-84.

5. Colocho G, Graham WP 3rd, Greene AE, Matheson DW, Lynch D. Human amniotic membrane as a physiologic wound dressing. Arch Surg 1974;109(3):370-3.

6. Talmi YP, Sigler L, Inge E, Finkelstein Y, Zohar Y. Antibacterial properties of human amniotic membranes. Placenta 1991;12(3):285-8.

7. Shimazaki J, Shinozaki N, Tsubota K. Transplantation of amniotic membrane and limbal autograft for patients with recurrent pterygium associated with symblepharon. $\mathrm{Br} \mathrm{J}$ Ophthalmol 1998;82(3):235-40.

8. Trelford JD, Trelford-Sauder M. The amnion in surgery, past and present. Am J Obstet Gynecol 1979;134(7)-833-45.

9. Tseng SC, Prabhasawat P, Lee SH .Amniotic membrane transplantation for conjunctival surface reconstruction. Am.J.Ophthalmol. 1997;124(6):765-74.

10. Meller D, Pires RT, Mack RJ, Figueiredo F, Heiligenhaus A, Park WC, et al. Amniotic membrane transplantation for acute chemical or thermal burns. Ophthalmology 2000 May 107(5) :980-9; discussion 990.

11. Pires RTF, Pires JLT, Tseng SCG. Transplante de membrana amniótica para reconstrução da superfície corneana e conjuntival . Arq Bras Oftalmol. 1999;62(3) : 340-50.

12. Muraine M, Descargues G, Franck O, Villeroy F, Toubeau D, Menguy E, et al.[Amniotic membrane graft in ocular surface disease .Prospective study with 31 cases]. J Fr Ophtalmol. 2001;;24(8) : 798-812. French.

13. Shimazaki J, Yang HY, Tsubota K, Amniotic membrane transplantation for ocular surface reconstruction in patients with chemical and thermal burns. Ophthalmology. 1997; 104(12) :2068-76.Comment in: Ophthalmology. 2000;107(3):411-2.

14. Tseng SC, Prabhasawat P, Barton K, Gray T, Meller D. Amniotic membrane transplantation with or without limbal allografts for corneal surface reconstruction in patients with limbal stem cell deficiency. Arch Ophthalmol 1998;116(4):431-41. 
15. Prabhasawat P, Barton K, Burkett G, Tseng SC. Comparison of conjunctival autografts, amniotic membrane grafts and primary closure for pterygium excision. Ophthalmology 1997;104(6):974-85.

16. Ma DH, See LC, Liau SB, Tsai RJ. Amniotic membrane graft for primary pterygium :comparison with conjunctival autograft and topical mitomycin $\mathrm{C}$ treatment. $\mathrm{Br} \mathrm{J}$ Ophthalmol 2000;84(9):973-8.

17. Lee SH, Tseng SC. Amniotic membrane transplantation for persistent ephithelial defects with ulceration. Am J Ophthalmol 1997;123(3): 303-12.

18. Chen HJ, Pires RT, Tseng SC. Amniotic membrane transplantation for severe neurotrophic corneal ulcers . Br J Ophthalmol 2000,84(8):826-33.

19. Honavar SG, Bansal AK, Sangwan VS, Rao GN. Amniotic membrane transplantation for ocular surface reconstruction in Stevens-Johnson Syndrome. Ophthalmology .2000; 107(5): 975-9.
20. Pires RT, Tseng SC, Parabhasawat P, Puangsricharern V, Maskim SL, Kim JC, Tan DT. Amniotic membrane transplantation for symptomatic bullous keratopathy. Arch Ophthalmol $1999 ; 117(10): 1291-7$.

21. Haraguchi DKM, Gomes JAP, Sato EH, Novo NF, Freitas D, et al. Uso de transplante de membrana amniótica no tratamento da ceratopatia bolhosa . Arq Bras Oftalmol. 2003; 66(2):121-4.
ENDEREÇO PARA CORRESPONDÊNCIA:
Eduardo Conforti de Oliveira
Av. Pinheiro Machado, 1283
CEP 18705-370 - Avaré - SP
Tel: (15) 8114-9120/(14) 3732.1151 ou HOSBOS
Tel: (15) 3212-7000 (Centro de Estudos)
E-mail : duconforti@hotmail.com 